\title{
A Behavior Based Trust Model for Grid Security
}

\author{
Vivekananth.P \\ Lecturer , IT Dept, \\ St Joseph college of engineering and \\ technology, Dar-Es-Salaam,Tanzania
}

\begin{abstract}
A Grid Computing system is a geographically distributed system with different domains, sharing resources. Security plays very important role in any internet system, specifically in Grid computing system. Since all the resources are shared in grid computing system, trust relationship is very crucial. Different domains have different policies in rendering their resources. They expect that the resources must be used by adhering to their policies.A security model must be made which shows the behavior conformity. The model must concentrate on behavior of entities in different domains, in different contexts. The trust model should satisfy the resource providers and the users. There are different trust models suggested by different research scholars. This paper proposes a model which ensures behavior conformity in grid computing.
\end{abstract}

\section{INTRODUCTION}

Trust can be thought of as firm belief in an entity to act dependably, securely and reliably in a specific context [1]. Trust depends upon the entities, time and context. For example an entity A wants to use the resources of $\mathrm{B}$, if the resource is a file then the required trust level will be lower. But if $\mathrm{A}$ wants to execute his code in B's machine then the required trust level will be higher. The code even may attempt to corrupt the whole system.

Behavioral trust can be defined as fulfilling the expectation of others. Behavioral trust can be classified as direct trust and indirect trust. It is impossible for any one to evaluate all aspects of an entity in all contexts to make trust decisions. So any entity has to depend on reputations of an entity by other entities. Trust can be the combination of direct trust evolved out of direct contacts and reputation trust from other known entities. In fact direct trust is also to be updated after each contact and experience. It is vital to provide a satisfactory dynamic trust model, which can assure behavior conformity. The trust model improves Grid security. This paper after analyzing some existing trust models, proposes a new trust model.

\section{RELATED WORK}

There are many papers focusing on trust models. The various trust models are briefly reviewed. In reference [1] trust reputation model is given. This model is developed by considering experience and reputation information. The total trust is evaluated from Agent direct trust and recommender trust which is depicted in figure 1 .

Direct trust degree td of any entity A with respect to context $\mathrm{c}$ is given by the formula

$$
\begin{aligned}
& \exists \operatorname{td} \varepsilon \mathrm{E}, \forall \text { se } \varepsilon \mathrm{s},(\mathrm{se}=\max (\mathrm{s})) \Rightarrow \mathrm{td}=\mathrm{e} \quad \text { where } \\
& \quad \mathrm{S}=(\mathrm{Svg}, \mathrm{Sg}, \mathrm{Sb}, \mathrm{Svb}) .
\end{aligned}
$$

E set of experiences; td direct trust degree; $\mathrm{S}$ set of levels of trust; $\mathrm{Svg}=$ very good, $\mathrm{Sg}=$ good, $\mathrm{Sb}=$ bad, $\mathrm{Svb}=$ very bad.

Recommender trust degree rtd of the entity A will be obtained by taking the mod of absolute value of members in the set. Ta $=\mathrm{Tvg}$ $\cup \mathrm{Tg} \cup \mathrm{Tb} \cup \mathrm{Tvb}$ where $\mathrm{T}$ represents set of experiences.

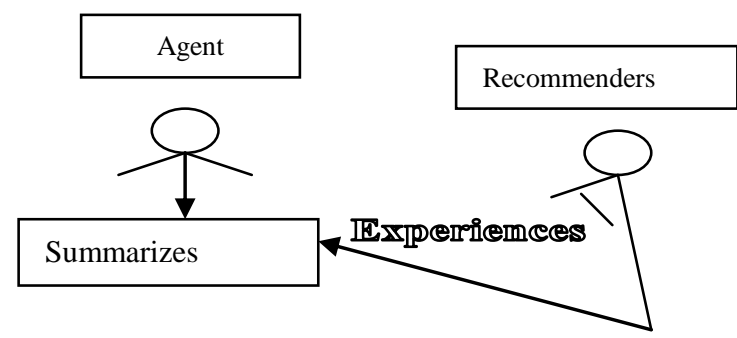

Figure 1 The trust model

In their paper [2] Farag Azzedin and Muthucumaru Maheswaran propose behavior trust model. They suggest that Trust Level is based upon past experience and specific context.

In this model Grid is divided into Grid domains. Resource and client domains are associated with each Grid Domain. Trust agents maintain Direct Trust table and Reputation Trust table. These two tables are used for calculating Total Trust. Weights are given for each direct and reputation trust relationships. Weightage given for direct trust relationship is greater than indirect trust relationship. It is assumed that trust decays with time and a decaying function is used to address this aspect.

In referenceb [3] Sanshan Song and Kai Hwang propose a fuzzy logic based behavior trust model . Trust worthiness is guaranteed by previous job experience and defense capabilities of resource sites.

In their paper [4] Justin R.D.Dyson,Nathan E. Griffiths suggest a trust framework model which enables users to execute their job in a reliable manner. Quality of service is the main aspect of this model. In this model, they use user-agents and resourceagents. Trust in a particular agent is represented by weight varying from 0 to 1.Trust is initially set to value according to agent's disposition. Trust is altered after the first transaction.

Gui Xiaolin,Xie Bing[5] suggests a behavior based trust model. They discuss about direct trust and indirect trust. Direct trust represents direct trust relationship and indirect trust is based on 
recommendation. Here accepting the recommendation from any entity depends on the individual policy. An entity may accept recommendation from the entity which it knows already. Here one attenuation function is assumed which decreases with time. If overall trust is obtained by any entity then the resource will be provided to that entity.

Baolin Ma,Jizhou Sun [6] talk about trust model based on reputation. In this model both direct and indirect trust are calculated by using reputation. $\mathrm{Ra}(\mathrm{b})$ denoted Reputation of $\mathrm{b}$ by a. $\mathrm{Ra}(\mathrm{b}, \mathrm{dir}), \mathrm{Ra}(\mathrm{b}$,indir) both are found. Direct trust is calculated and the value of direct trust is used to find the value of indirect trust.

\section{COMPARATIVE STUDY}

We have taken a scenario to compare the trust models proposed in [5]. and [6].

At time $t$, user A wants to run a job in the resource grid111 in windows domain. System prescribes if the overall trust is greater than or equal 3 the resource may be provided to A, other wise the request is rejected. In this example two domains are considered windows domain and the Linux domain. [Table1, Table2].

Table 1 Reputation Table

\begin{tabular}{|l|l|l|l|}
\hline 1. Windows & 2. Linux \\
\hline Resource & Trust value & Resource & $\begin{array}{l}\text { Trust } \\
\text { value }\end{array}$ \\
\hline Grid111 & 4 & Grid 247 & 2 \\
\hline 112 & 3 & 248 & 2 \\
\hline 113 & 4 & 249 & 2 \\
\hline 114 & 2 & 250 & 4 \\
\hline 115 & 3 & & \\
\hline
\end{tabular}

Table 2 ; Trust Relations between Domains

\begin{tabular}{|l|l|l|}
\hline Domain & Windows & Linux \\
\hline Windows & 0.5 & 0.25 \\
\hline Linux & 0.25 & 0.5 \\
\hline
\end{tabular}

According to the first model [5] the calculation of trust is as follows

In windows domain reputation

$0.25 * 3+0.25 * 4+0.25+2+0.25 * 3=3$

In Linux domain

$0.25 * 2+0.25 * 2+0.25 * 2+0.25 * 4=2.5$

The over all trust will be $\mathrm{T}=3 * 0.5+0.25 * 2.5=2.215$.

The over all trust is less then 3 . So the resource grid111 is rejected in the first model.

According to the second model [6] :

$\mathrm{M} \& \mathrm{~N}$ are windows and linux domain.

$\mathrm{Vn}(\mathrm{b})=$ Direct reliable value of entity $\mathrm{b}$ in domain $\mathrm{N}$.

$\operatorname{Tm}(\mathrm{N})=$ domain $\mathrm{N}$ 's credit value in $\mathrm{M}$.

$\mathrm{Tu}(\mathrm{N})=$ feed back from other domain

Trust $\mathrm{T}=\operatorname{Vn}(\mathrm{b})[\alpha \operatorname{Tm}(\mathrm{N})+(1-\alpha) \operatorname{Tm}(\mathrm{u}) * \mathrm{Tu}(\mathrm{N}) / \mathrm{x}]$

Where $\operatorname{Tm}(\mathrm{u})^{*} \mathrm{Tu}(\mathrm{N}) / \mathrm{x}$ is reputation from other domain.

$\alpha$ is taken as 0.5 where $\alpha$ is the weight factor.

$\mathrm{T}=4(0.5 * 0.25+0.5 * 2.5)=5.5$

$\mathrm{T}>3$

So the resource can be provided to user A.

The second model gives more weightage to direct trust (same domain). So there is variation in the result. The user of windows domain demands a resource in the same domain which has also the reputation of 4 . So the resource can be provided to him

\section{PROPOSED MODEL:}

A new trust model will be enhanced from these models. The total trust will be calculated by direct trust and indirect trust. Both the trust will be evaluated by reputations. There will be tracking module, which will keep track of behavior. Based on experiences with the entities, an entity trust level will be increased or decreased. There can be a penalty factor, which can be levied for malicious behaviors. The trust factor between two entities may depend on penalty, context and time. The penalty will be higher if the misbehavior creates heavy harm. Other wise the penalty will be low. Based on this experience the trust will be updated. The penalty factor can be a number between $0 \& 1$.

The model is explained as follows. [fig 2].

Direct trust DT

Reputation trust RT

Total trust $\mathrm{T}$

Required Trust $\mathrm{T}_{\text {min }}$ 


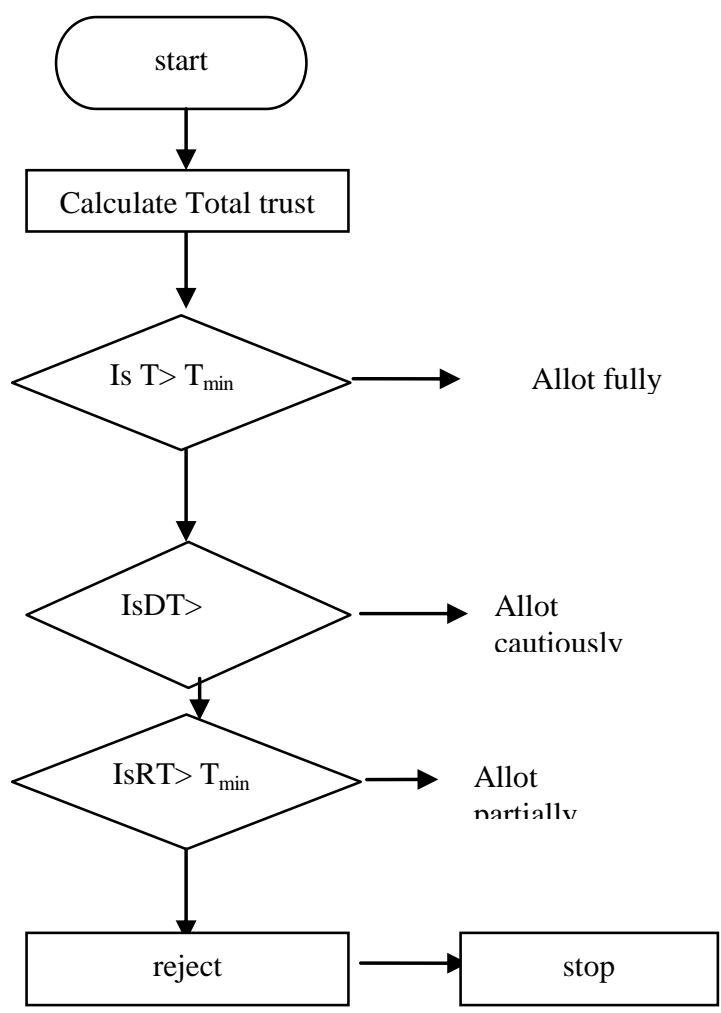

Figure 2 Proposed model

\section{CONCLUSION}

In this paper different trust models in grid security system have been analyzed and an enhanced behavior trust model is proposed. Here the feedback from past experience is taken and the penalty factor is considered.

\section{REFERENCES}

[1] Alfarez Abdul-Rahman, Stephen Hailes. Supporting Trust in Virtual Communities. International Conferences on System Sciences-2000

[2] Farag Azzedin and Muthucumaru Maheswaran. Evolving and Managing Trust in Grid Computing Systems. Canadian Conference on Electrical Computer Engineering-2002

[3] Shanshan Song ,Kai Hwang.Trusted Grid Computing with Security Assurance and Resource Optimization. International Conference on Parallel and Distributed Computing Systems-2004

[4] Justin R.D.Dyson, Nathan E.Griffiths. Trusting Agents for Grid Computing.IEEE International Conference on Systems, Man and Cybernetics-2004

[5] Gui Xiaolin,Xie Bing.Study on the Behavior based Trust Model in Grid Security System.IEEE International Conference on Services Computing-2004

[6] Boolin Ma,Jizhou Sun. Reputation-based Trust Model in Grid Security System. Journal of Communication and Computer,ISSNI548-7709,USA-2006.

If the total trust is greater than the required trust then the resource is allocated. We are trying to improve the proposed model further. 